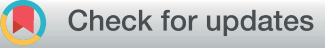

Cite this: J. Anal. At. Spectrom., 2017, 32,2053

Accepted 20th July 2017

DOI: $10.1039 / \mathrm{c} 6 \mathrm{ja00373g}$

rsc.li/jaas

\section{Comment on "Laser produced plasma diagnosis of carcinogenic heavy metals in gallstones" by $M$. A. Gondal, M. A. Shemis, A. A. I. Khalil, M. M. Nasr and B. Gondal, JAAS, 2016, 31, 506}

\author{
Andrey M. Popov, (D) * Timur A. Labutin (D) and Sergey M. Zaytsev \\ The selection of analytical lines to determine $\mathrm{Pb}, \mathrm{Cr}, \mathrm{Cd}, \mathrm{Hg}$, and $\mathrm{Ni}$ in gallstones in the recently published \\ paper with the above title is criticized. Also, the unreliable calibration of their spectrometer is demonstrated \\ as a reason for incorrect assignment of spectral lines.
}

The accurate quantitative detection of traces is undoubtedly the main challenge for wide analytical application of Laser-Induced Breakdown Spectroscopy (LIBS). In this regard, the paper by Gondal et al. ${ }^{1}$ has attracted our attention due to extremely low detection limits for the main "bad" heavy metals, such as lead, cadmium, mercury, etc. The authors declared the linearity of calibration curves in the range of 100 ppt to 1 ppm (see Fig. 4 in the paper ${ }^{1}$ ). For example, in the considered study $0.7 \mathrm{ppb}$ of mercury in a gallstone was "successfully" determined by LIBS, while the use of laser ablation ICP-MS, which is significantly more sensitive than LIBS, allowed reaching a limit of detection of 6.0 and $7.8 \mathrm{ppb}$ for mercury determination in a tooth ${ }^{2}$ and calcium carbonate, ${ }^{3}$ respectively. After reading the paper, we have found a number of serious systematic errors that raised doubts about the correctness of the authors' conclusions. We listed the main criticisms of the paper below beginning with the analytical lines used by the authors.

(1) Cadmium line Cd I $291.5 \mathrm{~nm}$. The authors wrote on page 509 that lines were identified with the use of the NIST Database. However, according to $i^{4}$ there are no atomic cadmium lines within the range of 290-295 $\mathrm{nm}$ ! Moreover, this line is absent in Kurucz's Database ${ }^{5}$ and in Optical Emission Lines of the Elements by Payling and Larkins. ${ }^{6}$ It should be noted that the strongest cadmium lines within the available spectral window $(250-850 \mathrm{~nm})$ are at 298.06, 346.62, 361.05, 508.58, 643.85 and $734.57 \mathrm{~nm}$ in accordance with the NIST Database. The only one of them (Cd I $298.06 \mathrm{~nm}$ ) was observed by the authors. Since the fulfillment of local thermodynamic equilibrium is stated (Section "Temperature and electron density calculations"), the intensity of the line at $643.85 \mathrm{~nm}\left(E_{\mathrm{upper}}=7.342 \mathrm{eV}\right)$ should be, at least, close to that of the line at $298.06 \mathrm{~nm}\left(E_{\text {upper }}=8.105 \mathrm{eV}\right)$ under the plasma conditions calculated by the authors for a 400 ns delay $\left(T=17200 \mathrm{~K}\right.$ and $\left.n_{\mathrm{e}}=3 \times 10^{18} \mathrm{~cm}^{-3}\right)$. The line at

Department of Chemistry, Lomonosov Moscow State University, Leninskie Gory 1-3, Moscow, 119234, Russia.E-mail: popov@laser.chem.msu.ru
$643.85 \mathrm{~nm}$, in fact, appears neither in Fig. 2 (ref. 1) nor in Fig. $3 .^{1}$ Therefore, the assignment of the lines at $298.06 \mathrm{~nm}$ and especially at $291.5 \mathrm{~nm}$ to cadmium is unreasonable.

(2) Lead line $P b I 368.3 \mathrm{~nm}$. Although the authors determined the lead content in two samples with this line (see Table 1 (ref. 1)), it is not visible in the spectra given in Fig. 2 (ref. 1) and $3 .^{1}$ Moreover, the strongest $\mathrm{Pb} \mathrm{I}$ lines at $283.30 \mathrm{~nm}$ and $405.78 \mathrm{~nm}$ belonging to the same multiplet ${ }^{3} \mathrm{P} \rightarrow{ }^{3} \mathrm{P}$ are absent in the spectra. The authors identified a lead line at $363.5 \mathrm{~nm}$ (upper spectrum in Fig. $2,{ }^{1}$ inset), but there are no lead lines in the vicinity of $363.5 \mathrm{~nm} .{ }^{4-6}$ One can be perplexed by the fact that there is the weak line Pb II $839.6 \mathrm{~nm}$ in Fig. 2 (ref. 1) for the first gallstone sample, while this line has disappeared in the spectrum of the second gallstone (Fig. 3 (ref. 1)). The opposite is the case for the weak line $\mathrm{Pb}$ II $719.4 \mathrm{~nm}$. It should be stressed that there are no other ionic or atomic lines of lead in the presented spectra. This fact implies that lead was not detected in the samples because all of the mentioned lines would be visible with different intensities in the spectra otherwise. We should also pay attention to incorrect spectroscopic notations in Table $1 .^{1}$ Since there are no doublet states (multiplicity $=2$ ) for atomic lead, the total angular moment $J$ in the LS-coupling case should be an integer (neither $1 / 2$ nor $3 / 2$ ) due to the integer values of the total spin moment $S$. According to the NIST Database ${ }^{4}$ the line $\mathrm{Pb} \mathrm{I}$ $368.3 \mathrm{~nm}$ belongs to the transition $\left[6 \mathrm{~s}^{2} 6 \mathrm{p} 7 \mathrm{~s}\right]{ }^{3} \mathrm{P}_{0} \rightarrow\left[6 \mathrm{~s}^{2} 6 \mathrm{p}^{2}\right]^{3} \mathrm{P}_{1}$.

(3) Chromium line CrI $425.4 \mathrm{~nm}$. We compared the parts of gallstone spectra presented by the authors in the insets of Fig. 2a (ref. 1) and 3a (ref. 1) in one picture for convenience (see our Fig. 1). Firstly, a red shift of the peak designated as Cr I 425.4 line in Fig. 1a relative to the tick at $426 \mathrm{~nm}$ is clearly seen. Secondly, the authors presented a very similar spectrum of the second gallstone with a quite different wavelength calibration (Fig. 1b).

The peak designated as K II $430.9 \mathrm{~nm}$ line (Fig. 1b) was shifted to the red relative to the tick at $431 \mathrm{~nm}$ ! It is necessary to mention that the corresponding transition has a very high excitation potential (23.514 eV (ref. 4)) as well as the O II line at 
a)

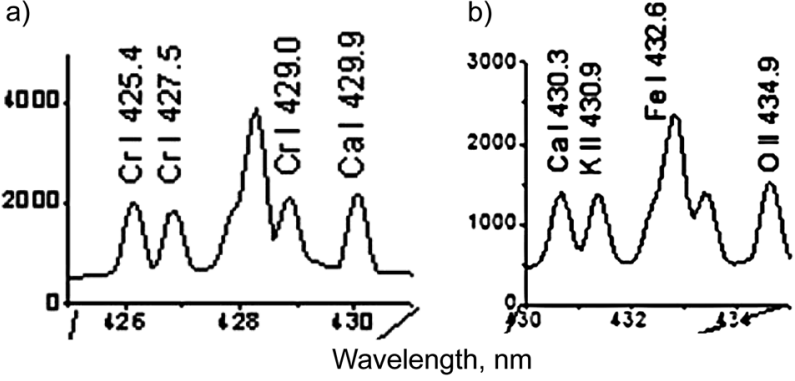

Fig. 1 Spectra of gallstone samples \#1 (a) and \#2 (b) taken from insets of Fig. $2 a$ and $3 a$ in the paper by Gondal et al., ${ }^{1}$ respectively. They demonstrate the incorrect wavelength calibration and line identification

the $434.9 \mathrm{~nm}$ (25.849 eV (ref. 4)); and these lines can barely be observed at the plasma temperature of $T \approx 1.5 \mathrm{eV}$. Thus, an obvious problem with the calibration of the spectrometer resulted in a misinterpretation of the spectra. We have applied a spectral modeling technique described earlier by us $^{7}$ to clarify this issue. A static model of homogeneous plasma under LTE conditions, accounting for broadening mechanisms, was used. Gallstones mainly consist of cholesterol or bilirubinate with inclusions of phosphates and carbonates and the major elements are carbon, oxygen, calcium, nitrogen and phosphorus. The calcium content can vary from $0.2 \%$ to $29.2 \%{ }^{8-10}$ We have taken the minimal Ca content of 0.2 mass $\%$, Cr of 0.000065 mass\% (close to the highest value in the calibration plot, see Fig. 4 in the original paper ${ }^{1}$ ), the atomized mass equal to $100 \mathrm{ng}$ for convenience and plasma parameters defined by the authors for a delay of $400 \mathrm{~ns}\left(T=17200 \mathrm{~K}, n_{\mathrm{e}}=3 \times 10^{18}\right.$ $\mathrm{cm}^{-3}$ ). Spectroscopic data were retrieved from the NIST Database. ${ }^{4}$ We chose the width of the instrument function $(0.3 \mathrm{~nm})$ for the model spectra to obtain a resolution close to those experimentally observed. The simulated spectra of the mixture of $\mathrm{Ca}$ and $\mathrm{Cr}$ and $\mathrm{Cr}$ only in the range of 425-431 nm are shown in Fig. 2. The intensities of the $\mathrm{Cr} I$ lines are $\sim 10^{4}$ times lower than those of the Ca I lines. Note that the ratios between Ca I line intensities within the multiplet ${ }^{3} \mathrm{P} \rightarrow{ }^{3} \mathrm{P}$ are very close to the experimental data of the authors ${ }^{1}$ (see "different" spectral ranges in Fig. 1). It should also be mentioned that the intensity ratios of the $\mathrm{Cr}$ I lines within multiplet $z^{7} \mathrm{P} \rightarrow a^{7} \mathrm{~S}$ in the range of 425-430 nm (Fig. 2b) differed significantly from those of the original spectrum (Fig. 1). Therefore, we believe that only Ca I lines were actually observed.

(4) Nickel line Ni I $361.9 \mathrm{~nm}$. This non-resonant line $\left(E_{\text {lower }}=\right.$ $0.423 \mathrm{eV}$ (ref. 4)) is present in the spectrum of the second gallstone (Fig. 3 (ref. 1)) and is absent in the spectrum of the first gallstone (see the left inset of Fig. 2a (ref. 1)). Nevertheless, the authors used it to determine the nickel content in both gallstones. The fact that the authors did not observe any components of the resonant multiplet besides the line at $345.8 \mathrm{~nm}$ (Fig. 3 (ref. 1)) appears suspicious. For example, the strongest $\mathrm{Ni}$ I line at $341.476 \mathrm{~nm}$ (resonant multiplet $\left[3 \mathrm{~d}^{9}\left({ }^{2} \mathrm{D}\right) 4 \mathrm{p}\right]{ }^{3} \mathrm{~F} \rightarrow$ $\left.\left[3 \mathrm{~d}^{9}\left({ }^{2} \mathrm{D}\right) 4 \mathrm{~s}\right]{ }^{3} \mathrm{D}\right)$ near the line at $361.9 \mathrm{~nm}$ was not observed.

(5) Mercury line $\mathrm{Hg} I 366.3 \mathrm{~nm}$ belongs to the ${ }^{3} \mathrm{D} \rightarrow{ }^{3} \mathrm{P}$ multiplet. ${ }^{4}$ Most of its components (a doublet at $313 \mathrm{~nm}$ and
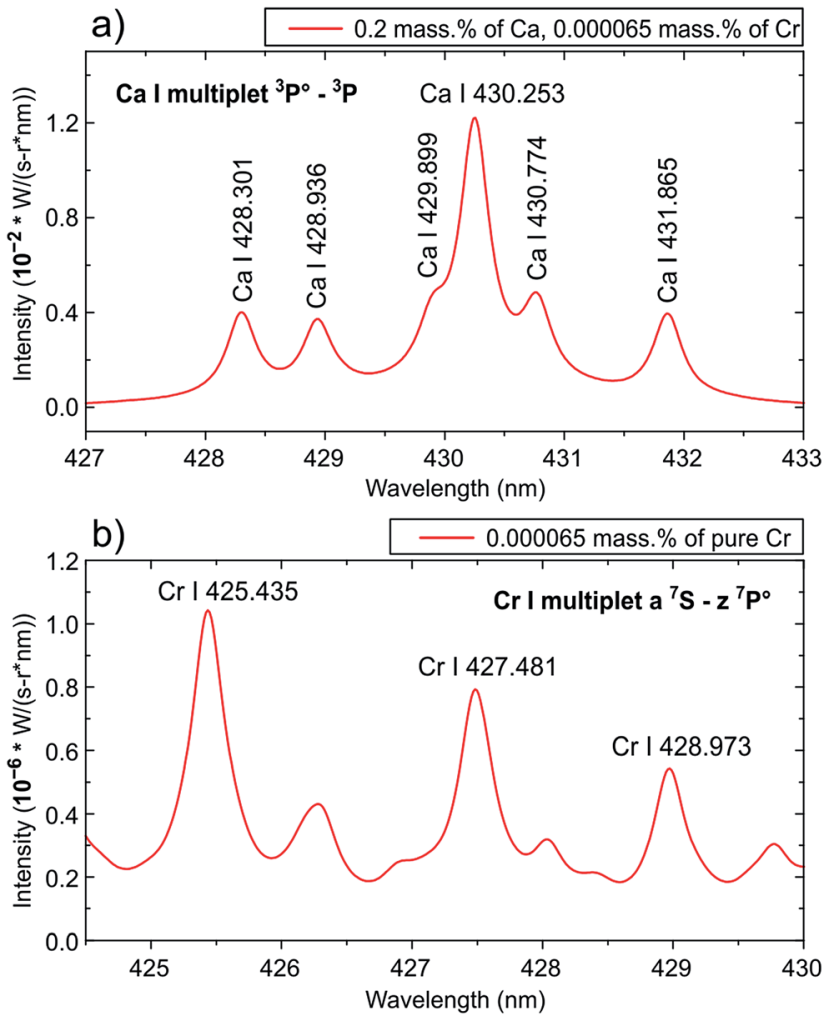

Fig. 2 (a) Simulated spectrum $\left(T=17200 \mathrm{~K}, n_{\mathrm{e}}=3 \times 10^{18} \mathrm{~cm}^{-3}\right)$ of the mixture of $\mathrm{Ca}(0.2$ mass $\%)$ and $\mathrm{Cr}(0.000065$ mass $\%)$; $\mathrm{Cr}$ lines are not observed. (b) Simulated spectrum of pure $\mathrm{Cr}(0.000065$ mass\%).

a line at $296.73 \mathrm{~nm}$ ) were not observed in the study (see Fig. 2 and 3 from the original paper ${ }^{1}$ ). The peak designated as $\mathrm{Hg}$ I $365.0 \mathrm{~nm}$ is blue-shifted relative to the tick at $365.0 \mathrm{~nm}$. Moreover, the intensity of the $\mathrm{Hg}$ I $365.02 \mathrm{~nm}$ line should be the highest within the multiplet. The line at $366.3 \mathrm{~nm}$, therefore, cannot be assigned to the above multiplet. Similar to the case of nickel, the line under discussion disappeared in the spectrum of the second gallstone (Fig. 3 (ref. 1)), but the authors somehow contrived to determine mercury in the stone. Another $\mathrm{Hg} I$ line at $435.8 \mathrm{~nm}$ is also observed in the spectrum of the first gallstone only (Fig. 2 (ref. 1)). Since the line belongs to triplet $\left.\left[5 \mathrm{~d}^{10} 6 \mathrm{~s} 7 \mathrm{~s}\right]\right]^{3} \mathrm{~S}$ $\rightarrow\left[5 \mathrm{~d}^{10} 6 \mathrm{~s} 6 \mathrm{p}\right]{ }^{3} \mathrm{P}$, other components at 546.07 and $404.66 \mathrm{~nm}$ should be visible too. Their absence in the spectra gives a reason to examine the correctness of mercury line assignment.

(6) As carbon is the main component of gallstones and bile, the authors ${ }^{1}$ had to observe carbon lines, which is not the case. For example, Singh et al. ${ }^{11}$ detected the strongest C I and C II lines in the UV range for LIBS analysis of gallstones. It should be mentioned that the authors have outlined the high sensitivity of their instrument in comparison of those used by Singh and colleagues.

(7) In addition, we pointed out some facts that supported our hypothesis about incorrect spectral calibration. The authors ascribed the two strongest lines within the range of 310-325 nm to Ca II for the first gallstone (Fig. 2a (ref. 1)) and to Na II for the second one (Fig. 3a (ref. 1)). Obviously, substitution of the Ca II lines by the Na II lines is incorrect due to (i) the observation of other ionic lines of calcium (for example, doublet near $395 \mathrm{~nm}$ ) 
and (ii) the extremely high excitation potential $\sim 41 \mathrm{eV}$ of $\mathrm{Na}$ II lines (by the way, their intensity ratios to resonant $\mathrm{Na}$ I lines at $589 \mathrm{~nm}$ are about $10^{-11}$ under determined plasma conditions). Moreover, there are no resonant Na I lines in the spectrum of the second gallstone. The last but not least observation concerns copper lines: the two resonant $\mathrm{Cu}$ I lines at 324 and $327 \mathrm{~nm}$ are not present in both spectra, while very weak ionic copper lines are observed. A similar case is observed for the lines O II 398.3, O II 407.6, P II 458.8 and K II 460.8.

The possible reason for the above-mentioned errors is the rule for line identification used by the authors: "The existence of the constituent elements was confirmed by identifying three finger-print spectral lines of each element or more" (page 509). It is well known that each of the elements has, at least, several hundreds of lines; therefore, a coincidence of three or more lines without consideration of their strength leads to an incorrect identification. That is why some simple spectroscopic rules mentioned by Cremers and Radziemski ${ }^{12}$ should be kept in mind: (i) the strongest lines of the main components must be present in emission spectra; (ii) observation of combinations of intense lines (multiplets): if one component is observed, the others should also be present; (iii) consideration of relative line intensities from spectral databases; and (iv) the presence of spectral lines of various ionized states depending on the experimental conditions. Currently, the NIST provides the calculation of multielement Saha-LTE spectra, ${ }^{4}$ which can help to implement the two last rules under particular experimental conditions. More information about the choice of the analytical lines can be found elsewhere. ${ }^{13}$

(8) A few remarks on plasma diagnostics should be added. Firstly, the authors erroneously associated electron density of a plasma with an optical one, which resulted in an absolutely incorrect conclusion: “...since McWhirter criteria are clearly satisfied which indicates that our plasma is thin..." (page 513 (ref. 1)). This criterion indicates whether LTE conditions exist, and its usage even as a test of the existence of LTE conditions is not recommended. ${ }^{14}$ Emission lines for plasma diagnostics should primarily be free of self-absorption and have well-known parameters. ${ }^{15}$ Secondly, since calcium is the main component of gallstones, the use of $\mathrm{Ca}$ II resonant lines have led to the overestimated values of electron density and temperature $(T=$ $17200 \mathrm{~K}, 3 \times 10^{18} \mathrm{~cm}^{-3}$ ) due to a profile distortion of the selfabsorbed lines. Thirdly, hypothetically the Na II lines (Fig. 7 (ref. 1)) may be used for plasma diagnostics, but their observation is doubtful on account of its high excitation potential $(>36 \mathrm{eV})$ in comparison with a plasma temperature of $\sim 1.5 \mathrm{eV}$. Also the energy axes in Fig. 7 (ref. 1) have the wrong dimension $-\mathrm{cm}^{-1}$ instead of eV. Finally, there are several contradictory statements regarding the profile of the Ca II $393.4 \mathrm{~nm}$ line: in the caption of Fig. 8 (ref. 1) and the sentence starting "Fig. 8 shows the..." the mentioned line profile is a Gaussian one, while in the sentence "The full width at half maximum (FWHM)..." the Lorentzian profile is stated.

Thus, we can conclude that the recovery of heavy metals in gallstones at extremely low levels apparently is not a fact, but is a result of erroneous experimental procedures, mostly wavelength calibration. Therefore, an explanation of strong correlation between the "analytical lines" and contents of analytes in standard samples (Fig. 4 (ref. 1)) is needed. Although, standard samples were used, there is no information on their type, composition, manufacturer, and analysis procedure. This seems to be important since the samples themselves may be a source of errors due to strong matrix effects. ${ }^{16}$ We suppose that the above-mentioned spectroscopic mistakes along with a lack of clarity of analytical aspects of calibration curve plotting are intolerable in a scientific journal on analytical spectroscopy.

\section{Acknowledgements}

The authors thank The Russian Foundation for Basic Researches (grant No. 15-33-70053_mol-a-mos) for the financial support for the software development needed to prepare the simulated spectra.

\section{References}

1 M. A. Gondal, M. A. Shemis, A. A. I. Khalil, M. M. Nasr and B. Gondal, J. Anal. At. Spectrom., 2016, 31, 506.

2 E. Hoffmann, H. Stephanowitz, E. Ullrich, J. Skole, C. Lüdke and B. Hoffmann, J. Anal. At. Spectrom., 2000, 15, 663.

3 A. Barats, C. Pécheyran, D. Amouroux, S. Dubascoux, L. Chauvaud and O. F. X. Donard, Anal. Bioanal. Chem., 2007, 387, 1131.

4 A. Kramida, Y. Ralchenko, J. Reader and NIST ASD Team, NIST Atomic Spectra Database (Ver. 5.3), National Institute of Standards and Technology, Gaithersburg, MD, 2015, http://physics.nist.gov/asd.

5 P. L. Smith, C. Heise, J. R. Esmond and R. L. Kurucz, Atomic Spectral Line Database from CD-ROM 23, ed. R. L. Kurucz, 1995, http:/www.pmp.uni-hannover.de/cgi-bin/ssi/test/kurucz/ sekur.html.

6 R. Payling and P. Larkins, CD for Optical Emission Lines of the Elements, John Wiley \& Sons, Ltd, 2000.

7 T. A. Labutin, S. M. Zaytsev and A. M. Popov, Anal. Chem., 2013, 85, 1985.

8 M. J. Whiting, V. Jarvinen and J. M. Watts, Gut, 1980, 21, 1077.

9 R. L. Baron, C. A. Rohrmann Jr, S. P. Lee, W. P. Shuman and S. A. Teefey, Am. J. Roentgenol., 1988, 151, 1123.

10 R. Schachler, T. Sauerbruch, U. Wosiewitz, J. Holl, D. Hahn, R. Denk, M. Neubrand and G. Paumgartner, Hepatology, 1988, 8, 925.

11 V. K. Singh, V. Singh, A. K. Rai, S. N. Thakur, P. K. Rai and J. P. Singh, Appl. Opt., 2008, 47, G38.

12 D. A. Cremers and L. J. Radziemski, Qualitative LIBS Analysis, in Handbook of Laser-induced Breakdown Spectroscopy, Wiley, 2nd edn, 2013, ch. 5, pp. 151-183.

13 N. B. Zorov, A. M. Popov, S. M. Zaytsev and T. A. Labutin, Russ. Chem. Rev., 2015, 84, 1021.

14 G. Cristoforetti, A. De Giacomo, M. Dell'Aglio, S. Legnaioli, E. Tognoni, V. Palleschi and N. Omenetto, Spectrochim. Acta, Part B, 2010, 65, 86.

15 C. Aragón and J. A. Aguilera, Spectrochim. Acta, Part B, 2008, 63, 893.

16 S. Zhang, M. He, Z. Yin, E. Zhu, W. Hang and B. Huang, J. Anal. At. Spectrom., 2016, 31, 358. 